Ciência Florestal, Santa Maria, v. 23, n. 1, p. 175-181, jan.-mar., 2013

ISSN 0103-9954

\title{
RELAÇÃO DO QUOCIENTE DO DIÂMETRO PELA ÁREA BASAL COM O AUTODESBASTE EM POVOAMENTO EQUIÂNEO DE Pinus taeda L.
}

\section{DIAMETER: THE BASAL AREA RATIO RELATION WITH THE SELF-THINNING IN Pinus taeda L. EVEN-AGED STAND}

\author{
Paulo Sérgio Pigatto Schneider ${ }^{1}$ César Augusto Guimarães Finger ${ }^{2}$ Paulo Renato Schneider ${ }^{3}$
}

\section{RESUMO}

Este trabalho teve por objetivo estudar o quociente do diâmetro pela área basal no momento da ocorrência do autodesbaste em povoamentos de Pinus taeda L., em vários espaçamentos, manejado em densidade completa. Para isso, foi utilizado o modelo Tang, relacionando o número de árvores por hectare com o diâmetro médio, a partir de dados de parcelas permanentes, medidas anualmente até os 18 anos de idade. Os resultados indicam que o modelo de autodesbaste proposto por Tang, apresentou ótima precisão e elevada eficiência, porém, com coeficiente angular preconizado por Tang de $(-3 / 2)$, tendo resultado um valor de -1,6523. No momento da ocorrência do autodesbaste, as árvores atingiram diâmetros de 14 e $17 \mathrm{~cm}$, nos espaçamento de $2 \times 2$ e $3 \times 2 \mathrm{~m}$, com áreas basais de 38,8 e $47,5 \mathrm{~m}^{2} \mathrm{ha}^{-1}$, respectivamente. Em todos os espaçamentos a área basal máxima foi de $78 \mathrm{~m}^{2} \mathrm{ha}^{-1}$, aos 18 anos de idade, com tendência ascendente. Nesses espaçamentos, os quocientes de diâmetro pela área basal no momento de ocorrência do autodesbaste foram de 0,36 e 0,37 , respectivamente.

Palavras-chave: autodesbaste; relação d:G; densidade; Pinus taeda.

\section{ABSTRACT}

This work undertakes to study de diameter: the basal area ratio at the moment of occurrence of self-thinning of Pinus taeda L. stand in some spacings, considering its full density. For that, the Tang model was used in order to relate the density of trees with the diameter, with data of permanent plots, measured annually until 18 years-old. The results indicate that self-thinning model for Tang presented excellent precision and high efficiency, however with distinct slope coefficient of the praised one for Tang $(-3 / 2)$ resulting a value of -1.6523. At the moment of occurrence of self-thinning, trees reached diameter equal to 14 and $17 \mathrm{~cm}$, in the spacing of $2 \times 2$ or $3 \times 2 \mathrm{~m}$, with basal area of 38.8 and $47.5 \mathrm{~m}^{2} \mathrm{ha}^{-1}$, respectively. In these spacings, the maximal basal area was of $78 \mathrm{~m}^{2} \mathrm{ha}^{-1}$, in 18 years-old, rising tendency. In these spacing, the diameter: the basal area ratio at the moment of occurrence of self-thinning was 0.36 and 0.37 , respectively.

Keywords: self-thinning; d:G ratio; density; Pinus taeda.

\section{INTRODUÇÃO}

O conhecimento do processo de competição intraespecífica, característica do crescimento e autodesbaste, é insuficiente para otimização do manejo dos povoamentos. A competição intraespecífica é um importante fator que afeta o tamanho médio das plantas em uma população e, por isso, tem sido muito estudada para descrever a relação entre o tamanho das plantas e a densidade da população, bem como a sua evolução nas árvores de Pinus taeda no tempo.

1. Engenheiro Florestal, Msc., Doutorando do Programa de Pós-graduação em Engenharia Florestal, Centro de Ciências Rurais, Universidade Federal de Santa Maria, Rua Alameda Sibipiruna 420, CEP 97095-660, Santa Maria (RS).psps@mail.ufsm.br

2. Engenheiro Florestal, Dr., Professor Associado do Departamento de Ciências Florestais, Centro de Ciências Rurais, Universidade Federal de Santa Maria, Av. Roraima, 1000, CEP 97105-900, Santa Maria (RS). finger@smail.ufsm.br

3. Engenheiro Florestal, Dr., Professor Titular do Departamento de Ciências Florestais, Centro de Ciências Rurais, Universidade Federal de Santa Maria, Av. Roraima, 1000, CEP 97105-900, Santa Maria (RS). paulors@smail.ufsm.br

Recebido para publicação em 5/10/2009 e aceito em 21/10/2011 
O conceito de densidade de manejo do povoamento é um processo de controle do crescimento do estoque inicial ou do estoque após desbastes para atender a objetivos específicos de manejo e de produção de matéria-prima. A determinação do nível apropriado do estoque de crescimento para um nível de povoamento é um processo complexo com envolvimento biológico, tecnológico e de fatores econômicos específicos para uma dada situação. $O$ processo requer a seleção de limites inferiores e superiores de estoque de árvores, referente a volume estocado, madeira estocada em uma determinada ocupação do sítio, visando a um adequado nível de produção, com um número de árvores desejado.

De acordo com Reineke (1933), em povoamento com densidade completa a relação entre o número de árvores por hectare $(\mathrm{N})$ e o diâmetro quadrático médio ( $\mathrm{dg}$ ) é linear em uma escala dupla logarítmica e que os parâmetros da função independem da idade e da qualidade do sítio. Postulou o autor que, a relação entre o número de árvores por hectare e o diâmetro médio quadrático em povoamentos sem tratamento é uma linha reta, com um valor do coeficiente angular constante de $-1,605$.

Del Rio et al. (2001) analisaram a relação do diâmetro médio e densidade populacional, com referência ao estabelecimento do autodesbaste em povoamento equiâneo não desbastado de Pinus silvestris, na Espanha. Os resultados indicaram que o autodesbaste seguiu uma linha côncava com o incremento do coeficiente angular e com o aumento da densidade do povoamento, assumindo a assíntota diretamente na linha de densidade máxima, com coeficiente angular igual a $-1,75$.

Zeide (1995) implementou a equação de densidade de Reineke com adição de um termo quadrático para representar uma curvatura na distribuição de densidade de árvores em função do diâmetro quadrático médio, sendo expressa por:

$$
\ln (N)=b_{0}-b_{1} \ln \left(d_{g}\right)+b_{2} \cdot\left(\ln \left(d_{g}\right)\right)^{2}
$$

A lei da potência -3/2, que indica $o$ autodesbaste, formulada por Yoda (1963), descreve a proporção máxima do número médio de árvores pelo tamanho de uma dada espécie, em decorrência da mortalidade é dependente da densidade. Essa relação entre o logarítmo do volume médio das árvores por hectare é linear, com coeficiente angular de $-1,5$. Essa linha de proporção se refere à máxima quantidade do número de árvores pelo tamanho, em que as plantas têm idêntica taxa de crescimento, para todos os locais, e o autodesbaste deve ocorrer quando as árvores tiverem uma completa ocupação do sítio. Essa lei da potência $-3 / 2$ pode ser escrita por: $\ln (w)=\ln (C)-(3 / 2) \cdot \ln (N)$, em que: $\mathrm{N}$ é o número de árvores por hectare, $\mathrm{w}$ é o peso médio, $\mathrm{C}$ é constante de proporcionalidade. Por conseguinte, o nome dado à lei surge da forma não linear da seguinte expressão: $\mathrm{w}=\mathrm{C} . \mathrm{N}^{-3 / 2}$.

Tang et al. (1994) estenderam a teoria do autodesbaste para povoamentos em densidade completa e subestocados constatando que o autodesbaste começa antes de o povoamento atingir a máxima densidade e que a taxa de autodesbaste incrementa com a densidade, atingindo maior valor em povoamento de densidade máxima. No modelo definido pelos autores, a lei do autodesbaste pode ser considerada um caso especial, pois permite obter para um diâmetro qualquer a densidade e a produção. Este modelo de autodesbaste é expresso por:

$$
\ln N(t)=\ln S_{f}-\frac{1}{\gamma} \ln \left[\left(D(t) / d_{0}\right)^{\beta \gamma}+\delta\right]
$$

Em que: $\mathrm{t}$ a idade do povoamento (ano), $\beta$ a taxa máxima de autodesbaste em densidade completa, $\mathrm{N}(\mathrm{t})$ a densidade no tempo $\mathrm{t}$ (número de árvores por hectare), $\mathrm{D}(\mathrm{t})$ o diâmetro médio das árvores no tempo $\mathrm{t}(\mathrm{cm}), \mathrm{S}_{\mathrm{f}} \mathrm{o}$ índice da máxima densidade, $\gamma$ o índice de autodesbaste, e $d_{o} o$ diâmetro básico padrão $(25 \mathrm{~cm})$. E, a constante $\delta$ foi determinada pela densidade inicial do povoamento $\left(\mathrm{N}_{1}\right)$ e o diâmetro médio inicial $\left(\mathrm{d}_{1}\right)$, pela seguinte fórmula: $\delta=\left(S_{f} / N_{1}\right)^{\gamma}-\left(d_{1} / d_{0}\right)^{\beta \gamma}$ em que, t é a idade do povoamento (ano); ainda para qualquer diâmetro, a densidade e a produção.

A lei do autodesbaste tem sido objeto de uso prático e de discussões acerca da sua eficiência por vários pesquisadores, como: Westoby (1981), West e Bourough (1983); Zeide (1985, 1987 e 1991); Smith e Hann (1986); Sterba e Monserud (1993); Yoda et al. (1963); Tang et al. (1994); Osawa e Allen (1993); Tang et al. (1995); Del Rio et al. (2001), entre outros.

Curtis (1982) utilizou o princípio e a equação de Reineke (1933) para descrever a relação entre a área basal $(\mathrm{G})$ e o diâmetro quadrático médio (dg) definindo para povoamentos de Pseudotsuga menziensii um valor para o coeficiente angular entre 0,45 e 0,5 , mas optando pelo valor 0,5 para determinar a densidade relativa e absoluta do povoamento. Recentemente, Reid (2006), com 
dados de plantações da Austrália e Nova Zelândia demonstrou o uso do quociente do diâmetro médio das árvores e a área basal do povoamento (d:G) para o manejo de florestas não industriais em pequenas propriedades rurais concluindo que o quociente é simples e prático para descrever o estoque de plantações de Eucalyptus, e que a manutenção desse quociente constante levou ao aumento da competição com o aumento da idade.

Assim, esse trabalho foi desenvolvido visando definir o valor do diâmetro médio e da área basal no momento do autodesbaste; o crescimento diamétrico e a ocorrência do autodesbaste; o momento de autodesbaste com base na proporção de diâmetro-área basal e a taxa de autodesbaste comparando-a com a lei da potência $-3 / 2$.

\section{MATERIAIS E MÉTODOS}

\section{Localização e caracterização da área de estudo}

Os dados foram levantados em florestas pertencentes à Klabin S.A., no município de Otacílio Costa, região fisiográfica do Planalto Central Catarinense (SANTA CATARINA, 1986).

O clima da região é do tipo "Cfb", mesotérmico, subtropical úmido, com verões frescos, não apresentando estação seca definida, segundo a classificação climática de Köppen, A precipitação média anual é de $1.808 \mathrm{~mm}$, distribuída em 124 dias, temperatura média anual de $17,8{ }^{\circ} \mathrm{C}$ (MOTTA et al., 1971).

O solo da área do experimento é classificado como latossolo bruno, alumínico TB, horizonte A húmico, textura argilosa e relevo suavemente ondulado.

Os dados foram medidos em experimento concebido no Modelo Nelder Modificado, instalado em 1988 e remedido periodicamente até a idade de 18 anos. Em cada unidade amostral foram medidas as circunferências a altura do peito e a altura das árvores. $\mathrm{O}$ delineamento estatístico foi em blocos ao acaso, com três repetições e nove tratamentos, sendo estes definidos pelo espaçamento inicial de plantio. Destes tratamentos foram utilizados os de: 1,5x1,0 (1,5 $\left.\mathrm{m}^{2}\right), 2,5 \mathrm{x} 1,0\left(2,5 \mathrm{~m}^{2}\right), 1,5 \times 2,0\left(3 \mathrm{~m}^{2}\right)$, $2,0 \times 2,0\left(4 \mathrm{~m}^{2}\right), 1,5 \times 3,0\left(4,5 \mathrm{~m}^{2}\right), 2,5 \times 2,0\left(5 \mathrm{~m}^{2}\right)$, $2,0 \times 3,0\left(6 \mathrm{~m}^{2}\right), 2,5 \times 3,0\left(7,5 \mathrm{~m}^{2}\right)$.

Os dados referentes ao número de árvores por hectare e diâmetro médio tiveram origem em parcelas permanentes localizadas em povoamentos implantados com vários espaçamentos, medidas periodicamente tendo originado, aproximadamente,
50 observações em cada espaçamento.

\section{Metodologia empregada}

O estudo da densidade e diâmetro e do autodesbaste foi realizado a partir do número de árvores por hectare e o correspondente diâmetro médio determinado nas unidades amostrais e ajustados com o procedimento NLIN, pelo método Gauss-Newton, no pacote SAS (1999). O modelo do autodesbaste empregado foi o proposto por Tang et al. (1994) expresso por:

$$
\ln N(t)=\ln S_{f}-\frac{1}{\gamma} \ln \left[\left(D(t) / d_{0}\right)^{\beta \gamma}+\delta\right]
$$

Sendo $\mathrm{t}$ a idade do povoamento (ano), $\beta$ a taxa máxima de autodesbaste em densidade completa, $\mathrm{N}(\mathrm{t})$ a densidade no tempo $\mathrm{t}$ (número de árvores por hectare), $\mathrm{D}(\mathrm{t})$ o diâmetro médio das árvores no tempo $\mathrm{t}(\mathrm{cm}), \mathrm{S}_{\mathrm{f}} \mathrm{o}$ índice da máxima densidade, $\gamma$ o índice de autodesbaste, e $d_{o} o$ diâmetro básico padrão $(25 \mathrm{~cm})$. E, a constante $\delta$ foi determinada pela densidade inicial do povoamento $\left(\mathrm{N}_{1}\right)$ e o diâmetro médio inicial $\left(\mathrm{d}_{1}\right)$, pela seguinte fórmula representativa, cujos valores esquemáticos aparecem no trabalho nas tabelas seguintes.

$$
\delta=\left(S_{f} / N_{1}\right)^{\gamma}-\left(d_{1} / d_{0}\right)^{\beta \gamma}
$$

O modelo de Tang foi calculado de forma independente para cada espaçamento inicial e, posteriormente, recalculado para os dados dos dois espaçamentos de maior densidade de plantio, respectivamente, $1,5 \times 1,0$ e 2,5x1,0 m.

Com os novos coeficientes, representando a máxima densidade foram geradas as curvas de densidade ajustadas para cada densidade de plantio permitindo identificar o estabelecimento do autodesbaste e a dimensão do diâmetro naquela ocasião.

Para verificar a precisão estatística dos modelos de densidade de árvores e diâmetro, foi determinado o coeficiente de determinação ajustado, erro padrão da estimativa, e o coeficiente de variação.

$\mathrm{Na}$ validação foram empregadas as estatísticas da tendência (Bias) e eficiência (E) dos modelos, recomendados por Palahi et al. (2002) e Cellini et al. (2002).

\section{RESULTADOS E DISCUSSÃO}

Os parâmetros estatísticos do modelo de autodesbaste de Tang gerados com o diâmetro e a 
densidade de árvores por hectare nos espaçamentos de plantio: $1,5 \times 1,0\left(1,5 \mathrm{~m}^{2}\right), 2,5 \times 1,0\left(2,5 \mathrm{~m}^{2}\right)$, 1,5x2,0 $\left(3 \mathrm{~m}^{2}\right), 2,0 \times 2,0\left(4 \mathrm{~m}^{2}\right), 1,5 \times 3,0\left(4,5 \mathrm{~m}^{2}\right)$, $2,5 \times 2,0\left(5 \mathrm{~m}^{2}\right), 2,0 \times 3,0\left(6 \mathrm{~m}^{2}\right), 2,5 \times 3,0\left(7,5 \mathrm{~m}^{2}\right)$, mostraram excelente ajuste e baixo erro padrão da estimativa, sem tendência, expressa no valor de bias inferior a 0,009 e alta eficiência, com valor superior a 0,999 , em todos os espaçamentos (Tabela 1 ).

Os dados da Tabela 1 mostraram, para todos os espaçamentos iniciais, que o modelo proposto por Tang apresentou um excelente ajuste e baixo erro da estimativa, com tendência muito pequena expressa no valor de bias inferior a 0,009 e alta eficiência, com valor superior a 0,999 , em todos os espaçamentos.

Analisando os valores do parâmetro $\beta$, que remete para a interpretação e comprovação da lei do autodesbaste, evidenciaram sua grande variação com o espaçamento de plantio, sem ter mantido uma tendência lógica de valores. Da mesma forma os parâmetros calculados diferiram do preconizado Tang et al. (1994) como igual a $-3 / 2$, para todas as situações de manejo e tratamento silvicultural. No espaçamento mais restrito $(1,5 \times 1,0 \mathrm{~m})$ o valor deste coeficiente $\beta$ foi igual 1,9578 e, por isto, não se pode afirmar sobre a universalidade da aplicação deste coeficiente angular como igual a $-3 / 2$ para todas as situações de manejo e tratamento silvicultural, como foi preconizado inicialmente na teoria definida por Tang et al. (1994).

A densidade de árvores por hectare para diferentes diâmetros médio calculada com o modelo de Tang nos espaçamentos definidos permitiu obter a curva decrescente da densidade de árvores por hectare, e a capacidade de tolerância fisiológica das árvores para se manterem vivas na população. Nessa condição passou a ocorrer o autodesbaste, porque, a partir deste ponto, as árvores passaram a não suportar mais a concorrência ocorrendo, normalmente, a morte das árvores mais dominadas da população.

A equação de densidade de diâmetro de Tang et al. (1994) padronizada com o diâmetro de referência de $25 \mathrm{~cm}$ e calculada com os dados observados nas parcelas permanentes dos espaçamentos $1,5 \times 1,0$ e 2,5 x 1,0 m, igualmente apresentou um ótimo ajuste, com um coeficiente de determinação de 0,99 , erro padrão da estimativa de 0,0948 e baixo coeficiente de variação, com valor de $1,17 \%$, comprovando a sua eficiência neste tipo de ajuste de dados. Com os dados de número de árvores por hectare e diâmetro médio observados nas parcelas permanentes de povoamento, nos espaçamentos de $1,5 \times 1,0$ e 2,5 x 1,0 m, foi ajustada a equação de densidade e diâmetro de Tang et al. (1994). Esta equação apresentou um ótimo ajuste, com um coeficiente de determinação de 0,99 , erro padrão de estimativa de 0,0948 e baixo coeficiente de variação.

$$
\begin{gathered}
\ln N=\ln 1494,5-\frac{1}{16,56} \ln \left[(d / 25)^{1,6523 * 16,56}\right. \\
\left.+\left(1494,5 / N_{1}\right)^{16,56}-\left(d_{1} / d_{0}\right)^{1,6523 * 16,56}\right]
\end{gathered}
$$

em que, d é o diâmetro médio das árvores, $\mathrm{d}_{1}$ é o diâmetro médio inicial, $\mathrm{d}_{0}$ é o diâmetro básico padrão $(25 \mathrm{~cm})$ e $\mathrm{N}_{1}$ a densidade de árvores iniciais.

Com o modelo foram gerados os dados de densidade de árvores por hectare pelo diâmetro médio do povoamento, obtendo-se uma curva decrescente da máxima densidade de árvores por hectare, que representa a máxima capacidade de tolerância fisiológica das árvores para se manterem vivas no habitat.

Esta tendência média foi representada na Figura 1, onde se pode observar a dimensão do

TABELA 1: Estatísticas do modelo de densidade e diâmetro de Tang, ajustado por espaçamento.

\begin{tabular}{|c|c|c|c|c|c|c|c|}
\hline \multirow{2}{*}{$\begin{array}{l}\text { Espaçamento } \\
\text { (m) }\end{array}$} & \multicolumn{3}{|c|}{ Parâmetros } & \multirow{2}{*}{$\mathrm{R}^{2}$} & \multirow{2}{*}{$\mathrm{S}_{\mathrm{yx}}$} & \multirow{2}{*}{ Bias } & \multirow{2}{*}{$\mathrm{E}$} \\
\hline & $S_{f}$ & $\beta$ & $\gamma$ & & & & \\
\hline $1,5 \times 1,0$ & 1378,3 & 1,9578 & 5,4411 & 0,9998 & 0,0595 & 0,0031 & 0,9997 \\
\hline $2,5 \times 1,0$ & 1354,9 & 1,6886 & 16,5602 & 0,9998 & 0,0549 & 0,0028 & 0,9997 \\
\hline $1,5 \times 2,0$ & 1213,0 & 1,7784 & 14,3297 & 0,9998 & 0,0495 & 0,0017 & 0,9999 \\
\hline $2,0 \times 2,0$ & 1406,8 & 1,3864 & 23,3919 & 0,9999 & 0,0274 & 0,0007 & 0,9999 \\
\hline $1,5 \times 3,0$ & 1595,9 & 1,9100 & 4,5000 & 0,9999 & 0,0558 & 0,0007 & 0,9999 \\
\hline $2,5 \times 2,0$ & 1352,8 & 1,2271 & 59,2456 & 0,9998 & 0.0423 & 0,0016 & 0,9998 \\
\hline $2,0 \times 3,0$ & 1401,0 & 1,2000 & 54,7276 & 0,9999 & 0,0276 & 0,0015 & 0,9998 \\
\hline $2,5 \times 3,0$ & 1159,9 & 1,7000 & 7,5600 & 0,9999 & 0,1014 & 0,0099 & 0,9998 \\
\hline
\end{tabular}

TABLE 1: Statistical results of the size-density model per the diameter of Tang, adjusted by spacing.

Fonte: Schneider (2008). 
diâmetro médio quando iniciou o autodesbaste, e ainda verificar que esse diâmetro variou, consideravelmente, com a densidade de árvores de plantio.

O momento exato da ocorrência do inicio da mortalidade de árvores (Figura 2), para um valor de diâmetro médio em um determinado número de árvores por hectare, correspondente aos espaçamentos iniciais testados, mostrou que o autodesbaste ocorreu em momentos diferentes com valores de diâmetros distintos, que aumentam numa relação direta com o espaçamento inicial, traduzindo em maior valor de diâmetro.

Quando o povoamento implantado em espaçamento de $2 \times 2$ ou $3 \times 2 \mathrm{~m}$ o autodesbaste ocorreu no momento em que o diâmetro médio atingiu valores de 14 e $19 \mathrm{~cm}$, respectivamente. A partir desse momento se estabeleceu uma expressiva concorrência e, por consequência, mortalidade de árvores, permitindo inferir que a execução de desbaste deveria ocorrer antes do início da mortalidade natural, evitando-se perdas de

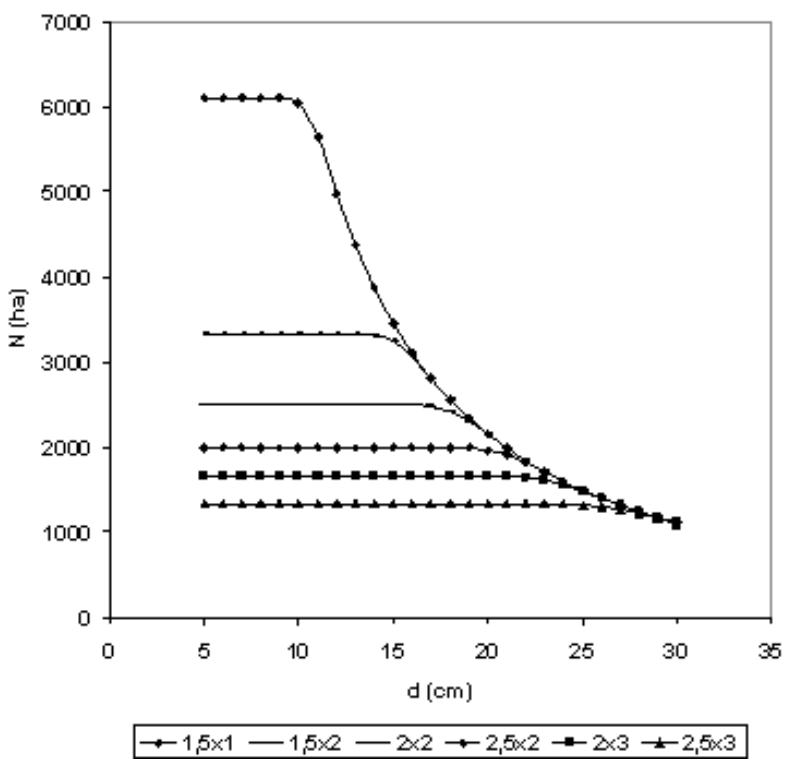

Fonte: Schneider (2008).

FIGURA 1: Tendências do autodesbaste, definido pela densidade de árvores por hectare em função do diâmetro médio, obtidas com o modelo proposto por Tang et al. (1994).

FIGURE 1: Self-thinning tendencies, defined by the density of trees per hectare in function of the average diameter, obtained from the model proposed by Tang et al. (1994). produção de madeira viva.

Nestas condições a relação do quociente do diâmetro médio pela área basal por hectare no momento de ocorrência do autodesbaste, representado na Figura 3, mostrou que o valor desse quociente variou significativamente com o espaçamento inicial de implantação do povoamento, onde nos espaçamentos de $2 \times 2$ e $3 \times 2 \mathrm{~m}$, os valores foram iguais a 0,36 e 0,40 .

A área basal por hectare calculada com o diâmetro médio de cada espaçamento e o número de árvores estimado com o modelo de Tang (Figura 4) mostrou que esta cresceu numa relação direta com o diâmetro médio da população, porém, em níveis estratificados segundo a densidade de plantio numa relação inversa com o espaço vital disponível às árvores. Em todos os espaçamentos utilizados os valores tenderam a aproximar-se da área basal máxima, acima de $78 \mathrm{~m}^{2} /$ ha, porém, mantendo a tendência de incremento ascendente.

O desenvolvimento do quociente do diâmetro médio pela área basal em todos os espaçamentos testados resultou em valores com grande variação (entre 0,3 a 2,0), observados a

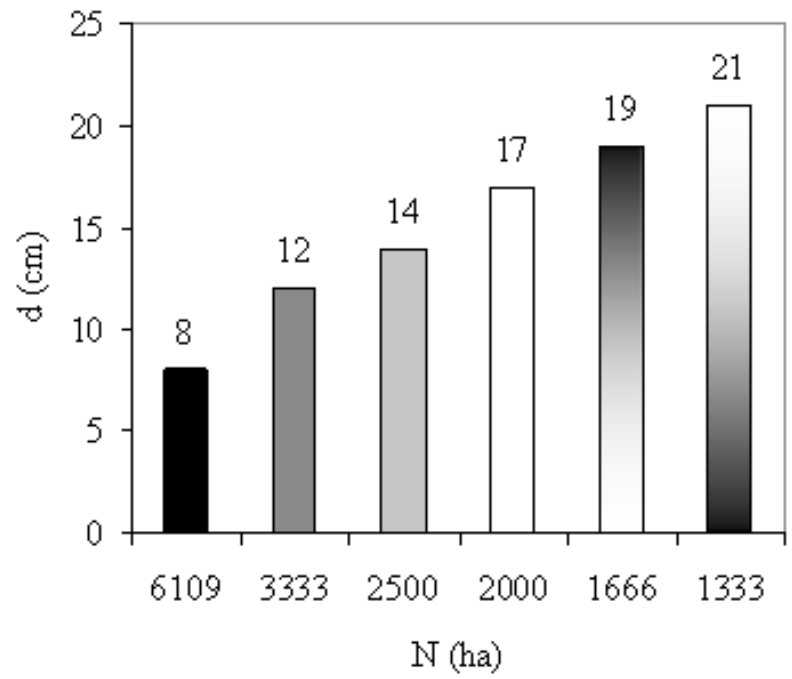

$1,5 \mathrm{x} 1 \square 1,5 \mathrm{x} 2 \square 2 \mathrm{x} 2 \square 2,5 \mathrm{x} 2 \square 2 \mathrm{x} 3 \square 2,5 \mathrm{x} 3$

FIGURA 2: Diâmetro médio do povoamento no momento da ocorrência do autodesbaste em relação do número de árvores por hectare do espaçamento inicial.

FIGURE 2: Average diameter of the stand at the moment of the occurrence of selfthinning in relation to the number of trees per hectare in the initial spacing. 
partir dos espaçamentos reduzidos aos mais amplos (Figura 5). Esse quociente descreveu uma curva com forma exponencial negativa em relação ao diâmetro médio, estabelecendo diferenças em níveis, tendo ocorrido sua estabilização em torno do valor 0,5 ,

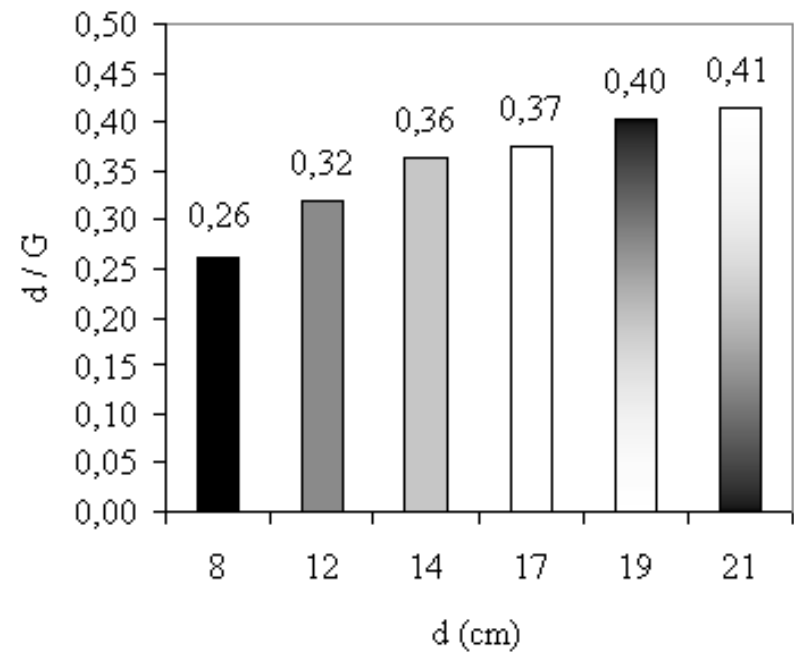

1,5x1 $\square$ 1,5x2 $\square 2 \mathrm{x} 2 \square 2,5 \mathrm{x} 2 \square 2 \mathrm{x} 3 \square 2,5 \mathrm{x} 3$

FIGURA 3: Quociente do diâmetro pela área basal no momento da ocorrência do autodesbaste por espaçamento inicial.

FIGURE 3: Diameter: the basal area ratio at the moment of occurrence of self-thinning per initial spacing.

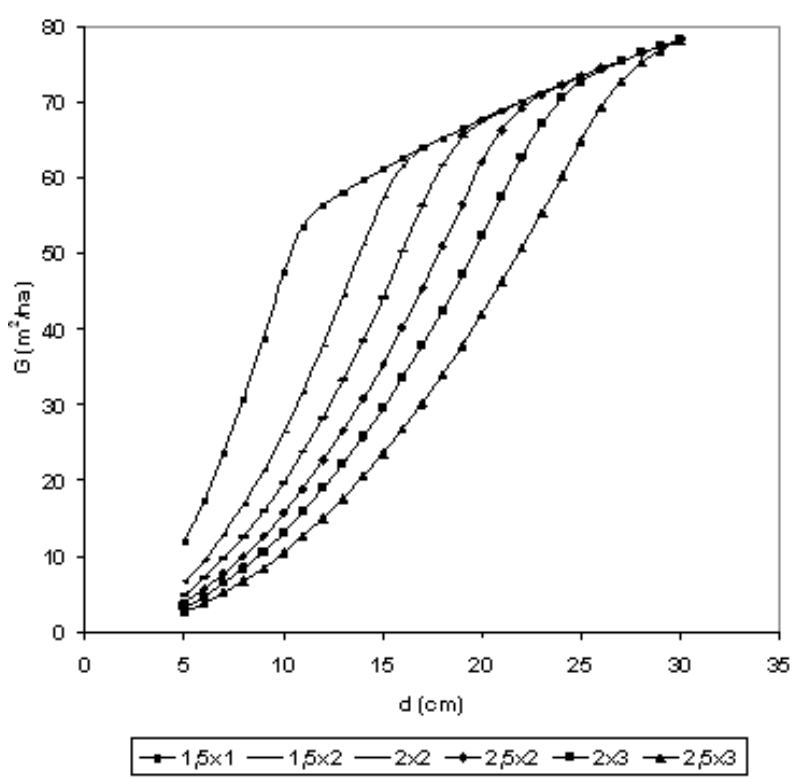

FIGURA 4: Desenvolvimento da área basal em relação ao diâmetro por espaçamento inicial.

FIGURE 4: Development of the basal area in diameter relation per initial spacing. encontrado, aproximadamente, no momento em que as curvas se aproximaram da curva do espaçamento mais reduzido, ou seja, com espaço vital de $1,5 \mathrm{~m}^{2}$ por ocasião do plantio.

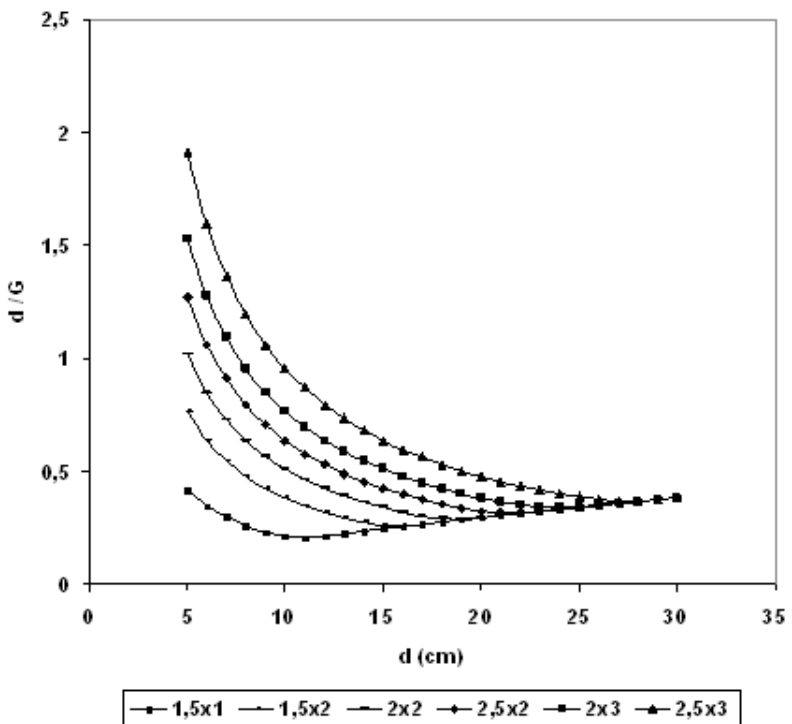

FIGURA 5: Quociente do diâmetro pela área basal em relação ao diâmetro por espaçamento inicial.

FIGURE 5: Diameter: the basal area ratio in diameter relation per initial spacing.

\section{CONCLUSÕES}

O modelo de autodesbaste de Tang apresenta ótima precisão estatística e elevada eficiência, com coeficiente angular de $-1,6523$, distinto do valor $-3 / 2$ prescrito para lei de autodesbaste;

$\mathrm{O}$ valor do diâmetro médio no momento da ocorrência do autodesbaste na população varia com a densidade de plantio das árvores, alcançando valores de 14 e $17 \mathrm{~cm}$, nos espaçamentos de 2 x 2 ou $3 \times 2 \mathrm{~m}$, respectivamente;

A relação entre o diâmetro médio pela área basal no momento de ocorrência do autodesbaste varia com o espaçamento inicial, com tendência a aproximar-se nos espaçamentos de 2 × 2 e $3 \times 2 \mathrm{~m}$, com valores de 0,36 e 0,37 , respectivamente;

Todos os espaçamentos utilizados tendem a aproximar-se da área basal máxima, em torno de $78 \mathrm{~m}^{2} / \mathrm{ha}$, aos 18 anos de idade, porém, nesta idade, ainda mostram diferentes tendências de incremento ascendente;

O quociente do diâmetro médio pela área basal numa dimensão temporal em todos 
os espaçamentos varia entre valores de 0,3 a 2,0, entre os espaçamentos reduzidos aos mais amplos, estabelecendo diferentes níveis, com estabilização em torno do quociente 0,5 , momento em que autodesbaste ocorre.

\section{AGRADECIMENTOS}

Os autores agradecem à KLABIN S.A., pela concessão dos dados dendrométricos, em especial aos engenheiros Antônio Maurício Moreira e Djalma Milles Chaves, responsáveis pela instalação e condução do experimento de espaçamento.

\section{REFERÊNCIAS BIBLIOGRÁFICAS}

CELLINI, J. M. et al. Modelos de perfil de tronco en Nothofagus pumilio (Polp, et Endl,) Krasser y su utilización en el cálculo del volumen total. Invest. Agr,: Sist. Recur. For., Madrid, n. 2, v. 11, p.245261, 2002.

CURTIS, R. O. A simple index of stand density for Douglas-fir. Forest Science, Bethesda, n.1, v.28, p.92-94, Mar. 1982.

DEL RIO, M.; MONTERO, G.; BRAVO, F. Analysis of diameter-density relationships and self-thinning in non-thinned even-aged Scots pine stands. Forest Ecology and Management. Amsterdan, v. 142, p. 79-87, Mar. 2001.

EMBRAPA. Sistema brasileiro de classificação de solos. Brasília: Embrapa, 1999, 412 p.

MOTTA, F. S.; BEIRSDORF, M. J. C.; GARCEZ, $R$. B. Zoneamento agrícola do Rio Grande do Sul e Santa Catarina: normas agro-climáticas. Pelotas: Ministério da Agricultura. 1971. 80 p.

OSAWA, A.; ALLEN, R. B. Allometric theory explains self-thinning relationships of mountain beech and Red pine. Ecology., n. 74, v. 4, p. $1020-$ 1032, Apr. 1993.

PALAHI, M.; MIIMA, J.; MONTERO, E. Standlevel yield model for scots pine (Pinus sylvestris) in north-east Spain. Invest. Agr. Sist. Recr. For., Madrid, v. 11, n. 2, p. 409-424, 2002.

REID, R. Diameter-basal area ratio as a practical stand density measure for pruned plantations. Forest Ecology and Management. Amsterdan, v. 233, p. 375-382, Sept. 2006.

REINEKE, L. H. Perfecting a stand-density index for even-aged forests. Canadian Agricultural Research. Ottawa, n. 46, p. 627-638, 1933.

SANTA CATARINA. Gabinete de Planejamento e Coordenação Geral. Subchefia de Estatística. Geografia e Informática. Atlas de Santa Catarina. Rio de Janeiro: Aerofoto Cruzeiro. 1986. 173 p.

SAS Institute Inc. SAS/STAT user's guide. Version 8 (computer manual). SAS Institute Inc., Cary. N.C. 1999.

SCHNEIDER, P. S. P. Autodesbaste e diagrama de manejo da densidade em povoamento de Pinus taeda L. 2008. 94 f. Dissertação (Mestrado em Engenharia Florestal) - Universidade Federal de Santa Maria, Santa Maria, 2008.

SMITH, N. J.; HANN, D. W. A growth model based on the self-thinning rule. Canadian Journal of Forest Research. New Westminster, v. 16, n. 2, p. 330-334, Apr. 1986.

STERBA, H.; MONSERUD, R. A. The maximum density concept applied to uneven-aged mixedspecies stands. Forest Science. Bethesda, v. 39, n. 3, p. 432-452, Aug. 1993.

TANG, S. et al, A growth and self-thinning model for pure even-aged stands: theory and applications. Forest Ecology and Management. Amsterdan, v. 70, p. 67-73, Dec. 1994.

TANG, S.; MENG, F. R.; MENG, C. H. The impact of initial stand density and site index on maximum stand density index and self-thinning index in a stand selfthinning model. Forest Ecology and Management. Amsterdan, v. 75, p. 61-68, June, 1995.

WEST, P. W.; BOUROUGH, C. J. Tree supervision and the self-thinning, Rule in a monoculture of Pinus radiata D, Don. Annals of Botany. Oxford, v. 52, p. 149-158, 1983.

WESTOBY, M. The place of the self-thinning rule in population dynamics. American Naturalist., v. 118, n. 4, p.581-587, Oct. 1981.

YODA, K.; KIRA, T.; OGAWA, H.; HOZUMI, K. Self-thinning in over crowed pure stands under cultivated and natural conditions. Jor. Biol. Osaka City Univ. Osaka, v. 14, p. 107-129, 1963.

ZEIDE, B. A relationship between size of trees and their number. Forest Ecology and Management. Amsterdan, v. 72, p. 265-272, Apr. 1995.

ZEIDE, B. Analysis of the $3 / 2$ power law of selfthinning. Forest Science. Bethesda, v. 33, n. 2, p. 17537, June, 1987.

ZEIDE, B. Self-thinning and stand density. Forest Science. Bethesda, v. 37, n. 2, p. 517-523, June, 1991.

ZEIDE, B. Tolerance and self-tolerance of trees. Forest Ecology and Management. Amsterdan, v. 13, p. 149-166, Nov. 1985. 